\title{
Endoscopic resection of a giant symptomatic Brunner's gland hamartoma of duodenal bulb
}

\author{
Umid Kumar Shrestha, Gopi Aryal
}

Nepal Mediciti Hospital, Bhaisepati, Lalitpur, Nepal

Keywords: Brunner's gland hamartoma; duodenal bulb; endoscopic resection

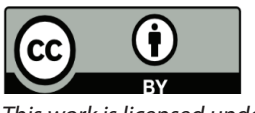

This work is licensed under a Creative Commons Attribution 4.0 Unported License.
Abstract

Brunnerss gland hamartoma is a very rare benign tumor arising from the Brunner's gland of duodenum and is usually asymptomatic, but may present with the symptom of duodenal obstruction or upper gastrointestinal bleeding due to ulceration from the tumor, requiring endoscopic or surgical resection. In our study, we report the case of a 57 year-old male who presented with pain over epigastrium, recurrent vomiting, black stool and dizziness with a lowering of hemoglobin up to $7.5 \mathrm{gm} / \mathrm{dl}$. The blood transfusion was done to raise the hemoglobin. The upper gastrointestinal endoscopy revealed a giant submucosal polypoidal mass with a thick short stalk in duodenal bulb causing almost complete obstruction of the lumen of duodenum and there was a superficial ulceration on the under-surface of the mass. The endoscopic ultrasound revealed a submucosal lesion arising from the echo layer three. The computed tomography of abdomen showed that the polypoidal mass was confined to the duodenal lumen with no significant lumphadenopathy and normal biliary and pancreas. The endoscopic polypectomy was attempted, but the lesion was too large to grab the polyp as a whole with the conventional snare. Hence, the repeated partial snare polypectomies were done, followed by submucosal dissection to ensure the complete removal of the mass. There were no complications after the procedure. The histopathology examination of the duodenal mass confirmed the diagnosis of Brunner's gland hamartoma and showed the presence of Helicobacter pylori as well. The endoscopic treatment of giant Brunner's gland hamartoma avoided the need of unnecessary more invasive surgical procedure.

\section{Introduction}

Brunner's gland hamartoma is a very rare benign tumor arising from the Brunner's gland of duodenum and is usually asymptomatic, but may present with the symptom of duodenal obstruction or upper gastrointestinal bleeding due to ulceration from the tumor, requiring endoscopic or surgical resection. ${ }^{1}$ Brunner's gland hamartomas account for $10.6 \%$ of all benign tumors of the duodenum, and have been found in $0.008 \%$ of a single series of 215,000 autopsies. ${ }^{2,3}$ We report a case of a patient with a large Brunner's gland hamartoma who presented with upper gastrointestinal bleeding and obstruction and was treated successfully by endoscopic removal of the tumor.
Correspondence-Author:

Umid Kumar Shrestha, MD, PhD, FACG

Head, Department of Gastroenterology, Hepatology and Endoscopy

Nepal Mediciti Hospital, Bhaisepati, Lalitpur, Nepal

Email: umidshrestha@gmail.com 


\section{Case Description}

Aln our study, we report the case of a 57 year-old male who presented with pain over epigastrium, recurrent vomiting, black stool and dizziness with a lowering of hemoglobin up to $7.5 \mathrm{gm} /$ dl. The blood transfusion was done to raise the hemoglobin. The upper gastrointestinal endoscopy revealed a giant submucosal polypoidal mass with a thick short stalk in duodenal bulb causing almost complete obstruction of the lumen of duodenum (figure 1) and there was a superficial ulceration on the under-surface of the mass

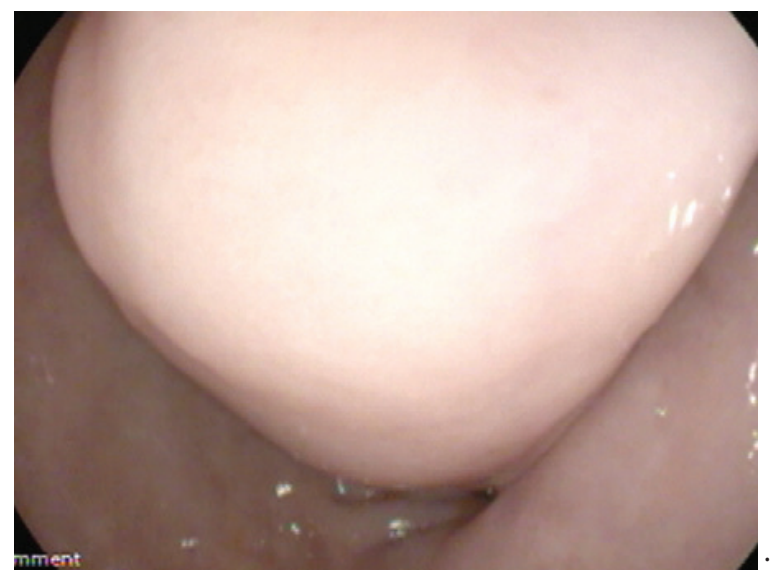

Figure1: Giant duodenal submucosal polypoidal mass (later on, histolopathology report showed Brunner's gland hamartoma) causing the almost complete obstruction of duodenum

The endoscopic ultrasound revealed a submucosal lesion arising from the echo layer three. The computed tomography of abdomen showed that the polypoidal mass was confined to the duodenal lumen with no significant lumphadenopathy and normal biliary and pancreas.

The endoscopic polypectomy was attempted, but the lesion was too large to grab the polyp as a whole with the conventional snare. Hence, the repeated partial snare polypectomies were done, followed by submucosal dissection to ensure the complete removal of the mass (figure 2); the mucosal defects were closed by hemoclips and hemostasis was maintained. There were no complications after the procedure. The endoscopic treatment avoided the need of unnecessary more invasive surgical procedure.

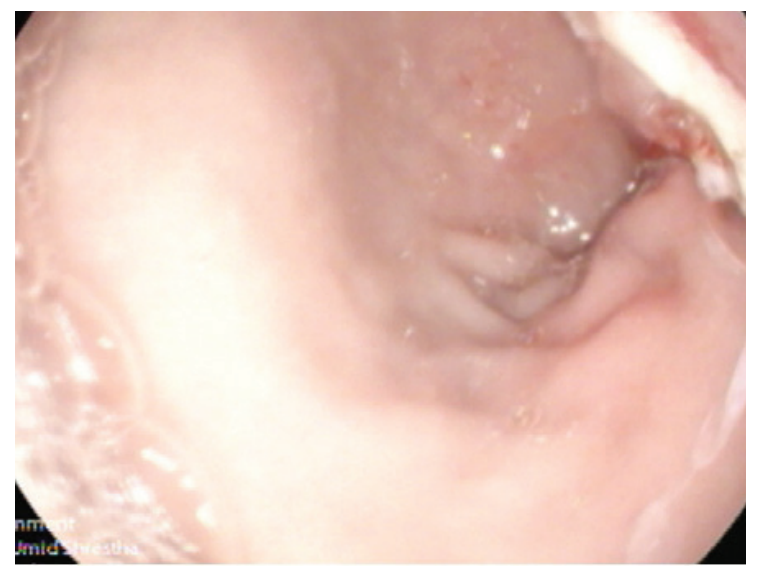

Figure 2: Endoscopic resection of the giant duodenal submucosalpolypoidal mass (later on, histolopathology report showed Brunner's gland hamartoma)
The histopathology examination of the duodenal mass confirmed the diagnosis of Brunner's gland hamartoma and showed the presence of Helicobacter pylori as well. He was started on proton pump inhibitor therapy and triple therapy regimen of Helicobacter pylori eradication was also given. His symptoms of pain over epigastrium, vomiting, melena and dizziness resolved after the endoscopic procedure. The patient was followed up at 3, 6, 12, 18 and 24 months. The hemoglobin was normalized throughout the period of follow up and a repeat upper gastrointestinal endoscopy did not show any evidence of recurrence or tumor remnants.

\section{Discussion}

Brunner's glands are located exclusively in the duodenum and consist of submucosal mucin-secreting glands. ${ }^{4}$ The $\mathrm{BGH}$ are generally located in the duodenal bulb $(57 \%)^{5,6}$ their size range from 0.7 to $12 \mathrm{~cm}$ in diameter with a mean of $4.0 \mathrm{~cm} .{ }^{7}$ Histologically, tumors of Brunner's glands are more consistent with hamartomas as opposed to neoplastic adenomas. The BGH consists of a mixture of ducts, acini, smooth muscle and adipose tissue ${ }^{6}$; it has no cellular atypia and is generally considered as non-malignant potential.,9 However, malignant potential has been described in one single case. ${ }^{6}$ As the Brunner's gland harmatomas enlarge, they typically develop into polypoid pedunculated masses. The exact pathogenesis is unknown, but it is hypothesized that they may be related to irritation of the local lining, chronic pancreatitis, Helicobacter pylori infection, or parasympathetic activity..$^{10}$ In our case there was a presence of Helicobacter pylori infection in the mass of $\mathrm{BGH}$, and hence, it seems that Helicobacter pylori infection could have contributed to the development of $\mathrm{BGH}$ in our patient. The common age of presentation of BGH is between 50 and 60 years and the incidence is similar in both genders. ${ }^{11}$ The common site of BGH is in the duodenal bulb (57\%), which may be due to the vascular damage and stress from gastrointestinal motility present here. However, the other sites where BGH can be seen are in the second (27\%) and third (7\%) parts of the duodenum. ${ }^{12}$

In our patient the Brunner's gland harmartoma had a giant polypoidal mass with a thick stalk, which caused near-complete obstruction, resulting in the obstructive symptoms of vomiting. The melena was due to the ulceration in the under-surface of the duodenal mass. The characteristic histological features of Brunner's gland harmartomas consist of an unusual mix of normal tissue and Brunner's glands, adipose cells, lymphoid cells, as well as ductal tissue and dysplasia is not observed..$^{13-15}$ The other possible differential diagnosis for duodenal mass lesions may be leiomyomas, adenomas, lipomas, adenocarcinomas, carcinoid tumors, lymphomas, leiomyosarcomas, pancreatic or ampullary tumors, and the lesions of Peutz-Jeghers syndrome.16 Treatment depends on presenting clinical features, size of the tumor and the possibility of malignancy. Asymptomatic Brunner's gland hamartomas may not require treatment as their neoplastic potential is low. ${ }^{3}$ However, treatment by removal of the mass is needed in case of large or symptomatic lesions that cause obstruction or hemorrhage. This can be done either endoscopically or surgically. Endoscopic treatment is preferred over surgical resection for pedunculated lesions; however, surgical treatment is needed if endoscopic methods fail, and if there is a suspicion of malignancy. In our patient, endoscopic resection was complete and successful despite the large size of the tumor; the mass was too large to grab into the conventional snare, and hence, the repeated piecemeal excision was done by partial snare polypectomies followed by submucosal dissection to complete the resection. 


\section{Conclusion:}

The endoscopic resection can be done for the treatment of even giant Brunnner's gland hamartoma of duodenal bulb in the hands of expert, avoiding the need of the unnecessary more invasive surgical procedure.

\section{References}

1. Sedano J, Swamy R, Jain K, et al. Brunner's gland hamartoma of the duodenum. Ann R Coll Surg Engl 2015; 97: e70-e72.

2. Nakabori T, Shinzaki S, Yamada T, et al. Atypical duodenal ulcer and invagination caused by a large pedunculated duodenal Brunner's gland hamartoma. Gastrointest Endosc 2014;79:679-680.

3. Jung $Y$, Chung IK, Lee $T H$, et al. Successful endoscopic resection of large pedunculated brunner's gland hamartoma causing gastrointestinal bleeding arising from the pylorus. Case Rep Gastroenterol 2013;7:304-307.

4. Gao YP, Zhu JS, Zheng WJ. Brunner's gland adenoma of duodenum: a case report and literature review World $J$ Gastroenterol 2004;10:2616-2617.

5. Levine JA, Burgart LJ, Batts K.P, et al. Brunner's gland hamartomas: clinical presentation and pathological features of 27 cases Am J Gastroenterol 1995;90:290-294.

6. Walden DT, Marcon NE. Endoscopic injection and polypectomy for bleeding Brunner's gland hamartoma: case report and expanded literature review Gastrointest Endosc 1998;47:403-407.

7. Hizawa K, Iwai K, Esaki M, et al. Endosonographic features of Brunner's gland hamartomas which were subsequently resected endoscopically Endoscopy 2002;34:956-958.

8. Attanoos R, Williams GT. Epithelial and neuroendocrine tumors of the duodenum Semin Diagn Pathol 1991;8:149162.
9. De Castella H. Brunner's gland adenoma. An unusual cause of intestinal bleeding Br J Surg 1966; 53:153-156.

10. Lee JH, Jo KM, Kim TO, et al. Giant Brunner's Gland Hamartoma of the Duodenal Bulb Presenting with Upper Gastrointestinal Bleeding and Obstruction. Clin Endosc 2016;49:570-574.

11. Lu L, LiR, Zhang G, et al. Brunner's gland adenoma of duodenum: report of two cases. Int J Clin Exp Pathol 2015;8:7565-7569.

12. Martinez MA, Zyromski NJ, Luz LP. An unusual case of large symptomatic Brunner's gland adenoma: endoscopic ultrasound imaging. Endosc Ultrasound 2015;4:266-267.

13. Gaspar JP, Stelow EB, Wang AY. Approach to the endoscopic resection of duodenal lesions. World J Gastroenterol 2016;22:600-617.

14. Cheung TT, Ip EW, Poon RT, et al. Brunner's gland adenoma: unusual cause of duodenal haemorrhage and obstruction. Hong Kong Med J 2013;19:460.e1-460.e2.

15. Stoos-Veic T, Tadic M, Aralica G. EUS-FNA of Brunner's gland hamartoma: a case report. Cytopathology 2013;24:194-196.

16. Sen R, Gupta V, Sharma N, et al. Brunner gland hamartoma masquerading as malignancy: a rare case report. Middle East J Dig Dis 2014;6:237-240. 